\title{
SHARP ENDPOINT INEQUALITY FOR MULTILINEAR LITTLEWOOD-PALEY OPERATOR
}

\author{
LANZHE LIU
}

\begin{abstract}
We establish a sharp inequality for multilinear Littlewood-Paley operator. As application, we obtain the weighted norm inequalities and $L \log L$ type endpoint estimate for the multilinear operator.
\end{abstract}

\section{Introduction and result}

Let $\psi$ be a function on $R^{n}$ which satisfies the following properties:

(1) $\int \psi(x) d x=0$,

(2) $|\psi(x)| \leq C(1+|x|)^{-(n+1)}$

(3) $|\psi(x+y)-\psi(x)| \leq C|y|(1+|x|)^{-(n+2)}$ when $2|y|<|x|$;

Let $m$ be a positive integer and $A$ be a function on $R^{n}$. We denote that $\Gamma(x)=\left\{(y, t) \in R_{+}^{n+1}:|x-y|<t\right\}$ and the characteristic function of $\Gamma(x)$ by $\chi_{\Gamma(x)}$. The multilinear Littlewood-Paley operator is defined by

$$
S_{\psi}^{A}(f)(x)=\left[\iint_{\Gamma(x)}\left|F_{t}^{A}(f)(y)\right|^{2} \frac{d y d t}{t^{n+1}}\right]^{1 / 2},
$$

where

$$
\begin{aligned}
& F_{t}^{A}(f)(x, y)=\int_{R^{n}} \frac{f(z) \psi_{t}(y-z)}{|x-z|^{m}} R_{m+1}(A ; x, z) d z, \\
& R_{m+1}(A ; x, y)=A(x)-\sum_{|\alpha| \leq m} \frac{1}{\alpha !} D^{\alpha} A(y)(x-y)^{\alpha},
\end{aligned}
$$

and $\psi_{t}(x)=t^{-n} \psi(x / t)$ for $t>0$. We write $F_{t}(f)(x)=f * \psi_{t}(x)$. We also define that

$$
S_{\psi}(f)(x)=\left(\iint_{\Gamma(x)}\left|F_{t}(f)(x)\right|^{2} \frac{d y d t}{t^{n+1}}\right)^{1 / 2},
$$

2000 MR Subject Classification: 42B20, 42B25.

Keywords: Multilinear Littlewood-Paley operator; Sharp estimate; BMO.

Received June 25, 2003. 
which is the Littlewood-Paley operator (see [12]).

Let $H$ be the Hilbert space $H=\left\{h:\|h\|=\left(\iint_{R_{+}^{n+1}}|h(t)|^{2} \frac{d y d t}{t^{n+1}}\right)^{1 / 2}<\infty\right\}$. Then for each fixed $x \in R^{n}, F_{t}^{A}(f)(x)$ and $F_{t}(f)(x)$ may be viewed as a mapping from $(0,+\infty)$ to $H$, and it is clear that

$$
S_{\psi}^{A}(f)(x)=\left\|\chi_{\Gamma(x)} F_{t}^{A}(f)(x, y)\right\| .
$$

Note that when $m=0, S_{\psi}^{A}$ is just the commutator of Littlewood-Paley operator (see [9]), while when $m>0$, it is non-trivial generalizations of the commutators. It is well known that multilinear operators are of great interest in harmonic analysis and have been widely studied by many authors (see [1-5]). In [8], authors establish a variant sharp estimate for the multilinear singular integral operators. The main purpose of this paper is to establish a sharp estimate for the multilinear Littlewood-Paley operator, then the weighted norm inequalities and the $L \log L$ type endpoint estimate for the multilinear operator are obtained by using the sharp estimate. We point out that some of our ideas come from [8] and [10]. First, let us introduce some notation (see [6] [7] [10]).

For any locally integrable function $f$, the sharp function of $f$ is defined by

$$
f^{\#}(x)=\sup _{x \in Q} \frac{1}{|Q|} \int_{Q}\left|f(y)-f_{Q}\right| d y,
$$

where, and in what follows, $Q$ will denote a cube with sides parallel to the axes, and $f_{Q}=|Q|^{-1} \int_{Q} f(x) d x$. It is well-known that

$$
f^{\#}(x)=\sup _{x \in Q} \inf _{c \in C} \frac{1}{|Q|} \int_{Q}|f(y)-c| d y .
$$

We say that $f$ belongs to $B M O\left(R^{n}\right)$ if $f^{\#}$ belongs to $L^{\infty}\left(R^{n}\right)$. For $0<r<\infty$, we denote $f_{r}^{\#}$ by

$$
f_{r}^{\#}(x)=\left[\left(|f|^{r}\right)^{\#}(x)\right]^{1 / r} .
$$

Let $M$ be the Hardy-Littlewood maximal operator, that is

$$
M f(x)=\sup _{x \in Q} \frac{1}{|Q|} \int_{Q}|f(y)| d y,
$$

we write that $M_{p} f=\left(M\left(f^{p}\right)\right)^{1 / p}$, for $k \in N$, we denote by $M^{k}$ the operator $M$ iterated $k$ times, i.e., $M^{1} f(x)=M f(x)$ and

$$
M^{k} f(x)=M\left(M^{k-1} f\right)(x) \text { when } k \geq 2 .
$$

Let $B$ be a Young function and $\tilde{B}$ be the complementary associated to $B$, we denote that, for a function $f$

$$
\|f\|_{B, Q}=\inf \left\{\lambda>0: \frac{1}{|Q|} \int_{Q} B\left(\frac{|f(y)|}{\lambda}\right) d y \leq 1\right\}
$$

and the maximal function by 


$$
M_{B} f(x)=\sup _{x \in Q}\|f\|_{B, Q}
$$

The main Young function to be using in this paper is $B(t)=t\left(1+\log ^{+} t\right)$ and its complementary $\tilde{\boldsymbol{B}}=\exp t$, the corresponding maximal denoted by $M_{L \log L}$ and $M_{\exp L}$. We have the generalized Holder's inequality (see [10])

$$
\frac{1}{|Q|} \int_{Q}|f(y) g(y)| d y \leq\|f\|_{B, Q}\|g\|_{B, Q}
$$

and the following inequality (in fact they are equivalent), for any $x \in R^{n}$

$$
M_{L \log L} f(x) \leq C M^{2} f(x)
$$

and the following inequalities, for all cube $Q$ any $b \in B M O\left(R^{n}\right)$

$$
\left\|b-b_{Q}\right\|_{\exp L, Q} \leq C\|b\|_{B M O}
$$

and

$$
\left|b_{2^{k+1} Q}-b_{2 Q}\right| \leq 2 k\|b\|_{B M O} .
$$

We denote the Muckenhoupt weights by $A_{p}$ for $1 \leq p<\infty$ (see [6]).

Now we state the results in this paper as following.

THEOREM 1. Let $D^{\alpha} A \in B M O\left(R^{n}\right)$ for all $\alpha$ with $|\alpha|=m$. Then for any $0<r<1$, there exists a constant $C>0$ such that for any $f \in C_{0}^{\infty}\left(R^{n}\right)$ and any $x \in R^{n}$,

$$
\left(S_{\psi}^{A}(f)\right)_{r}^{\#}(x) \leq C \sum_{|\alpha|=m}\left\|D^{\alpha} A\right\|_{B M O} M^{2} f(x) .
$$

Theorem 2. Let $1<p<\infty$ and $D^{\alpha} A \in B M O\left(R^{n}\right)$ for all $\alpha$ with $|\alpha|=m$, $w \in A_{p}$. Then $S_{\psi}^{A}$ is bounded on $L^{p}(w)$, that is

$$
\left\|S_{\psi}^{A}(f)\right\|_{L^{p}(w)} \leq C \sum_{|\alpha|=m}\left\|D^{\alpha} A\right\|_{B M O}\|f\|_{L^{p}(w)} .
$$

Theorem 3. Let $D^{\alpha} A \in B M O\left(R^{n}\right)$ for all $\alpha$ with $|\alpha|=m, w \in A_{1}$. Then there exists a constant $C>0$ such that for each $\lambda>0$,

$$
\begin{aligned}
w(\{x & \left.\left.\in R^{n}: S_{\psi}^{A}(f)(x)>\lambda\right\}\right) \\
& \leq C \sum_{|\alpha|=m}\left\|D^{\alpha} A\right\|_{B M O} \int_{R^{n}} \frac{|f(x)|}{\lambda}\left(1+\log ^{+}\left(\frac{|f(x)|}{\lambda}\right)\right) w(x) d x .
\end{aligned}
$$

As in [10], Theorem 2 and 3 follow from Theorem 1 and the boundedness of $S_{\psi}$ with $M$. So we only need to prove Theorem 1 .

\section{Some lemmas}

We begin with some preliminary lemmas. 
Lemma 1 (Kolmogorov, [7, p. 485]). Let $0<p<q<\infty$ and for any function $f \geq 0$. We define that

$$
\begin{gathered}
\|f\|_{W L^{q}}=\sup _{\lambda>0} \lambda\left|\left\{x \in R^{n}: f(x)>\lambda\right\}\right|^{1 / q}, \\
N_{p, q}(f)=\sup _{E}\left\|f \chi_{E}\right\|_{L^{p}} /\left\|\chi_{E}\right\|_{L^{r}}, \quad(1 / r=1 / p-1 / q)
\end{gathered}
$$

where the sup is taken for all measurable sets $E$ with $0<|E|<\infty$. Then

$$
\|f\|_{W L^{q}} \leq N_{p, q}(f) \leq(q /(q-p))^{1 / p}\|f\|_{W L^{q}} .
$$

Lemma 2 ([10, p. 165]). Let $w \in A_{1}$. Then there exists a constant $C>0$ such that for any function $f$ and for all $\lambda>0$,

$$
w\left(\left\{y \in R^{n}: M^{2} f(y)>\lambda\right\}\right) \leq C \lambda^{-1} \int_{R^{n}}|f(y)|\left(1+\log ^{+}\left(\lambda^{-1}|f(y)|\right)\right) w(y) d y .
$$

Lemma 3 ([3, p. 448]). Let $A$ be a function on $R^{n}$ and $D^{\alpha} A \in L^{q}\left(R^{n}\right)$ for all $\alpha$ with $|\alpha|=m$ and some $q>n$. Then

$$
\left|R_{m}(A ; x, y)\right| \leq C|x-y|^{m} \sum_{|\alpha|=m}\left(\frac{1}{|\tilde{Q}(x, y)|} \int_{\tilde{Q}(x, y)}\left|D^{\alpha} A(z)\right|^{q} d z\right)^{1 / q},
$$

where $\tilde{Q}(x, y)$ is the cube centered at $x$ and having side length $5 \sqrt{n}|x-y|$.

Lemma 4. Let $1<p<\infty, \quad 1<r \leq \infty, \quad 1 / q=1 / p+1 / r$ and $D^{\alpha} A \in$ $B M O\left(R^{n}\right)$ for all $\alpha$ with $|\alpha|=m$. Then $S_{\psi}^{A}$ is bound from $L^{p}\left(R^{n}\right)$ to $L^{q}\left(R^{n}\right)$, that is

$$
\left\|S_{\psi}^{A}(f)\right\|_{L^{q}} \leq C \sum_{|\alpha|=m}\left\|D^{\alpha} A\right\|_{B M O}\|f\|_{L^{p}}
$$

Proof. By Minkowski inequality and the condition of $\psi$, we have

$$
\begin{aligned}
S_{\psi}^{A}(f)(x) & \leq \int_{R^{n}} \frac{|f(z)|\left|R_{m+1}(A ; x, z)\right|}{|x-z|^{m}}\left(\int_{\Gamma(x)}\left|\psi_{t}(y-z)\right|^{2} \frac{d y d t}{t^{1+n}}\right)^{1 / 2} d z \\
& \leq C \int_{R^{n}} \frac{|f(z)|\left|R_{m+1}(A ; x, z)\right|}{|x-z|^{m}}\left(\int_{0}^{\infty} \int_{|x-y| \leq t} \frac{t^{-2 n}}{(1+|y-z| / t)^{2 n+4}} \frac{d y d t}{t^{1+n}}\right)^{1 / 2} d z \\
& \leq C \int_{R^{n}} \frac{|f(z)|\left|R_{m+1}(A ; x, z)\right|}{|x-z|^{m}}\left(\int_{0}^{\infty} \int_{|x-y| \leq t} \frac{t^{-2 n}}{(1+|y-z| / t)^{2 n+4}} \frac{d y d t}{t^{n+1}}\right)^{1 / 2} d z, \\
& \leq C \int_{R^{n}} \frac{|f(z)|\left|R_{m+1}(A ; x, z)\right|}{|x-z|^{m}}\left(\int_{0}^{\infty} \int_{|x-y| \leq t} \frac{2^{2 n+4} \cdot t^{1-n}}{(2 t+|y-z|)^{2 n+2}} d y d t\right)^{1 / 2} d z,
\end{aligned}
$$


noting that $2 t+|y-z| \geq 2 t+|x-z|-|x-y| \geq t+|x-z|$ when $|x-y| \leq t$ and

$$
\int_{0}^{\infty} \frac{t d t}{(t+|x-z|)^{2 n+2}}=C|x-z|^{-2 n}
$$

we obtain

$$
\begin{aligned}
S_{\psi}^{A}(f)(x) & \leq C \int_{R^{n}} \frac{|f(z)|}{|x-z|^{m}}\left|R_{m+1}(A ; x, z)\right|\left(\int_{0}^{\infty} \frac{t d t}{(t+|x-z|)^{2 n+2}}\right)^{1 / 2} d z \\
& =C \int_{R^{n}} \frac{|f(z)|}{|x-z|^{m+n}}\left|R_{m+1}(A ; x, z)\right| d z,
\end{aligned}
$$

thus, the lemma follows from [4] [5].

\section{Proof of Theorems}

We first prove Theorem 1.

Proof of Theorem 1. For $\tilde{x} \in R^{n}$, let $Q=Q\left(x_{0}, l\right)$ be a cube centered at $x_{0}$ and having side length $l$ such that $\tilde{x} \in Q$. It is suffice to prove for $f \in C_{0}^{\infty}\left(R^{n}\right)$ and some constant $C_{0}$, the following inequality holds:

$$
\left(\frac{1}{|Q|} \int_{Q}\left|S_{\psi}^{A}(f)(x)-C_{0}\right|^{r} d x\right)^{1 / r} \leq C M^{2} f(\tilde{x})
$$

Set $\quad \tilde{Q}=5 \sqrt{n} Q \quad$ and $\quad \tilde{A}(x)=A(x)-\sum_{|\alpha|=m} \frac{1}{\alpha !}\left(D^{\alpha} A\right)_{\tilde{Q}} x^{\alpha}$, then $R_{m}(A ; x, y)=$ $R_{m}(\tilde{A} ; x, y)$ and $D^{\alpha} \tilde{A}=D^{\alpha} A-\left(D^{\alpha} A\right)_{\tilde{Q}}$ for $|\alpha|=m$. We write, for $f_{1}=f \chi_{\tilde{Q}}$ and $f_{2}=f \chi_{R^{n} \backslash \tilde{Q}}$,

$$
\begin{aligned}
F_{t}^{A}(f)(x, y)= & \int \frac{R_{m+1}(A ; x, z)}{|x-z|^{m}} \psi_{t}(y-z) f(z) d z \\
= & \int \frac{R_{m+1}(\tilde{A} ; x, z)}{|x-z|^{m}} \psi_{t}(y-z) f_{2}(z) d z+\int \frac{R_{m}(\tilde{A} ; x, z)}{|x-z|^{m}} \psi_{t}(y-z) f_{1}(z) d z \\
& -\sum_{|\alpha|=m} \frac{1}{\alpha !} \int \frac{(x-z)^{\alpha} D^{\alpha} \tilde{A}(z)}{|x-z|^{m}} \psi_{t}(y-z) f_{1}(z) d z
\end{aligned}
$$

then

$$
\begin{aligned}
& \left|S_{\psi}^{A}(f)(x)-S_{\psi}^{\tilde{A}}\left(f_{2}\right)\left(x_{0}\right)\right| \\
& \quad=\left|\left\|\chi_{\Gamma(x)} F_{t}^{A}(f)(x, y)\right\|-\left\|\chi_{\Gamma\left(x_{0}\right)} F_{t}^{\tilde{A}}(f)\left(x_{0}, y\right)\right\|\right| \\
& \quad \leq\left\|\chi_{\Gamma(x)} F_{t}^{A}(f)(x, y)-\chi_{\Gamma\left(x_{0}\right)} F_{t}^{\tilde{A}}(f)\left(x_{0}, y\right)\right\|
\end{aligned}
$$


SHARP ENDPOINT INEQUALITY FOR MULTILINEAR LITTLEWOOD-PALEY OPERATOR

$$
\begin{aligned}
\leq & \left\|\chi_{\Gamma(x)} F_{t}\left(\frac{R_{m}(\tilde{A} ; x, \cdot)}{|x-\cdot|^{m}} f_{1}\right)(y)\right\|+\sum_{|\alpha|=m} \frac{1}{\alpha !}\left\|\chi_{\Gamma(x)} F_{t}\left(\frac{(x-\cdot)^{\alpha}}{|x-\cdot|^{m}} D^{\alpha} \tilde{A} f_{1}\right)(y)\right\| \\
& +\left\|\chi_{\Gamma(x)} F_{t}^{\tilde{A}}\left(f_{2}\right)(x, y)-\chi_{\Gamma\left(x_{0}\right)} F_{t}^{\tilde{A}}\left(f_{2}\right)\left(x_{0}, y\right)\right\| \\
\equiv & I(x)+I I(x)+I I I(x),
\end{aligned}
$$

thus,

$$
\begin{aligned}
& \left(\frac{1}{|Q|} \int_{Q}\left|S_{\psi}^{A}(f)(x)-S_{\psi}^{\tilde{A}}\left(f_{2}\right)\left(x_{0}\right)\right|^{r} d x\right)^{1 / r} \\
& \quad \leq\left(\frac{C}{|Q|} \int_{Q} I(x)^{r} d x\right)^{1 / r}+\left(\frac{C}{|Q|} \int_{Q} I I(x)^{r} d x\right)^{1 / r}+\left(\frac{C}{|Q|} \int_{Q} I I I(x)^{r} d x\right)^{1 / r} \\
& \quad \equiv I+I I+I I I .
\end{aligned}
$$

Now, let us estimate $I, I I$ and $I I I$, respectively. First, for $x \in Q$ and $y \in \tilde{Q}$, using Lemma 2, we get

$$
R_{m}(\tilde{A} ; x, y) \leq C|x-y|^{m} \sum_{|\alpha|=m}\left\|D^{\alpha} A\right\|_{B M O},
$$

thus, by Lemma 1 and the weak type $(1,1)$ of $S_{\psi}$ (see [9] [12]), we obtain

$$
\begin{aligned}
I & \leq C \sum_{|\alpha|=m}\left\|D^{\alpha} A\right\|_{B M O}|Q|^{-1} \frac{\left\|S_{\psi}\left(f_{1}\right) \chi_{Q}\right\|_{L^{r}}}{\left\|\chi_{Q}\right\|_{L^{r /(1-r)}}} \\
& \leq C \sum_{|\alpha|=m}\left\|D^{\alpha} A\right\|_{B M O}|Q|^{-1}\left\|S_{\psi}\left(f_{1}\right)\left(f_{1}\right)\right\|_{W L^{1}} \\
& \leq C \sum_{|\alpha|=m}\left\|D^{\alpha} A\right\|_{B M O}|\tilde{Q}|^{-1} \int_{\tilde{Q}}|f(y)| d y \\
& \leq C \sum_{|\alpha|=m}\left\|D^{\alpha} A\right\|_{B M O} M(f)(\tilde{x}) ;
\end{aligned}
$$

For $I I$, similar to the proof of $I$, we get

$$
\begin{aligned}
I I & \leq C \sum_{|\alpha|=m}|Q|^{-1} \frac{\left\|S_{\psi}\left(D^{\alpha} \tilde{A} f_{1}\right) \chi_{Q}\right\|_{L^{r}}}{\left\|\chi_{Q}\right\|_{L^{r /(1-r)}}} \leq C \sum_{|\alpha|=m}|Q|^{-1}\left\|S_{\psi}\left(D^{\alpha} \tilde{A} f_{1}\right)\right\|_{W L^{1}} \\
& \leq C \sum_{|\alpha|=m}|Q|^{-1} \int_{\tilde{Q}}\left|D^{\alpha} \tilde{A}(y)\right||f(y)| d y \leq C \sum_{|\alpha|=m}\left\|D^{\alpha} A\right\|_{\exp L, \tilde{Q}}\|f\|_{L \log L, \tilde{Q}} \\
& \leq C \sum_{|\alpha|=m}\left\|D^{\alpha} A\right\|_{B M O} M_{L \log L} f(\tilde{x}) \leq C \sum_{|\alpha|=m}\left\|D^{\alpha} A\right\|_{B M O} M^{2} f(\tilde{x}) ;
\end{aligned}
$$


Now let us estimate $I I I$. We write

$$
\begin{aligned}
\chi_{\Gamma(x)} F_{t}^{\tilde{A}}\left(f_{2}\right)(x, y)-\chi_{\Gamma\left(x_{0}\right)} F_{t}^{\tilde{A}}\left(f_{2}\right)\left(x_{0}, y\right) \\
=\int_{R^{n}}\left[\frac{1}{|x-z|^{m}}-\frac{1}{\left|x_{0}-z\right|^{m}}\right] \chi_{\Gamma(x)} \psi_{t}(y-z) R_{m}(\tilde{A} ; x, z) f_{2}(z) d z \\
\quad+\int \frac{\chi_{\Gamma(x)} \psi_{t}(y-z) f_{2}(z)}{\left|x_{0}-z\right|^{m}}\left[R_{m}(\tilde{A} ; x, z)-R_{m}\left(\tilde{A} ; x_{0}, z\right)\right] d z \\
\quad+\int\left(\chi_{\Gamma(x)}-\chi_{\Gamma\left(x_{0}\right)}\right) \frac{\psi_{t}(y-z) R_{m}\left(\tilde{A} ; x_{0}, z\right) f_{2}(z)}{\left|x_{0}-z\right|^{m}} d z \\
\quad-\sum_{|\alpha|=m} \frac{1}{\alpha !} \int\left[\frac{\chi_{\Gamma(x)}(x-z)^{\alpha}}{|x-z|^{m}}-\frac{\chi_{\Gamma\left(x_{0}\right)}\left(x_{0}-z\right)^{\alpha}}{\left|x_{0}-z\right|^{m}}\right] \psi_{t}(y-z) D^{\alpha} \tilde{A}(z) f_{2}(z) d z \\
=I I I_{1}+I I I_{2}+I I I_{3}+I I I_{4} .
\end{aligned}
$$

Note that $|x-z| \sim\left|x_{0}-z\right|$ for $x \in Q$ and $z \in R^{n} \backslash \tilde{Q}$. By Lemma 3 and the following inequality (see [7])

$$
\left|b_{Q_{1}}-b_{Q_{2}}\right| \leq C \log \left(\left|Q_{2}\right| /\left|Q_{1}\right|\right)\|b\|_{B M O}, \quad \text { for } Q_{1} \subset Q_{2},
$$

we know that, for $x \in Q$ and $z \in 2^{k+1} \tilde{Q} \backslash 2^{k} \tilde{Q}$,

$$
\begin{aligned}
\left|R_{m}(\tilde{A} ; x, z)\right| & \leq C|x-z|^{m} \sum_{|\alpha|=m}\left(\left\|D^{\alpha} A\right\|_{B M O}+\left|\left(D^{\alpha} A\right)_{\tilde{Q}(x, z)}-\left(D^{\alpha} A\right)_{\tilde{Q}}\right|\right) \\
& \leq C k|x-z|^{m} \sum_{|\alpha|=m}\left\|D^{\alpha} A\right\|_{B M O} ;
\end{aligned}
$$

For $I I I_{1}$, by the condition on $\psi$ and similar to the proof of Lemma 4 , we get

$$
\begin{aligned}
\left\|I I I_{1}\right\| & \leq \int_{R^{n} \backslash \tilde{Q}} \frac{\left|x-x_{0}\right||f(z)|}{|x-z|^{n+m+1}}\left|R_{m}(\tilde{A} ; x, z)\right| d z \\
& \leq \sum_{k=0}^{\infty} \int_{2^{k+1} \tilde{Q} \backslash 2^{k} \tilde{Q}} \frac{\left|x-x_{0}\right||f(z)|}{|x-z|^{n+m+1}}\left|R_{m}(\tilde{A} ; x, z)\right| d z \\
& \leq C \sum_{|\alpha|=m}\left\|D^{\alpha} A\right\|_{B M O} \sum_{k=1}^{\infty} k 2^{-k} M(f)(\tilde{x}) \\
& \leq C \sum_{|\alpha|=m}\left\|D^{\alpha} A\right\|_{B M O} M(f)(\tilde{x}) ;
\end{aligned}
$$

For $\mathrm{II}_{2}$, by the formula (see [3]):

$$
R_{m}(\tilde{A} ; x, z)-R_{m}\left(\tilde{A} ; x_{0}, z\right)=\sum_{|\beta|<m} \frac{1}{\beta !} R_{m-|\beta|}\left(D^{\beta} \tilde{A} ; x, x_{0}\right)(x-z)^{\beta}
$$


and Lemma 3, we have

$$
\begin{aligned}
\left|R_{m}(\tilde{A} ; x, z)-R_{m}\left(\tilde{A} ; x_{0}, z\right)\right| & \leq C \sum_{|\beta|<m} \sum_{|\alpha|=m}\left|x-x_{0}\right|^{m-|\beta|}|x-z|^{|\beta|}\left\|D^{\alpha} A\right\|_{B M O} \\
& \leq C \sum_{|\alpha|=m}\left\|D^{\alpha} A\right\|_{B M O}\left|x-x_{0}\right||x-z|^{m-1},
\end{aligned}
$$

thus, similar to the proof of Lemma 4

$$
\begin{aligned}
\left\|I I I_{2}\right\| & \leq \int_{R^{n} \backslash \tilde{Q}} \frac{\left|R_{m}(\tilde{A} ; x, z)-R_{m}\left(\tilde{A} ; x_{0}, z\right)\right|}{\left|x_{0}-z\right|^{m+n}}|f(z)| d z \\
& \leq C \sum_{|\alpha|=m}\left\|D^{\alpha} A\right\|_{B M O} \sum_{k=0}^{\infty} \int_{2^{k+1} \tilde{Q} \backslash 2^{k} \tilde{Q}} \frac{|Q|^{1 / n}}{\left|x_{0}-z\right|^{n+1}}|f(z)| d z \\
& \leq C \sum_{|\alpha|=m}\left\|D^{\alpha} A\right\|_{B M O} \sum_{k=1}^{\infty} 2^{-k} \frac{1}{\left|2^{k} \tilde{Q}\right|} \int_{2^{k} \tilde{Q}}|f(z)| d z \\
& \leq C \sum_{|\alpha|=m}\left\|D^{\alpha} A\right\|_{B M O} M(f)(\tilde{x}) ;
\end{aligned}
$$

For $I I I_{3}$, similar to the proof of Lemma 4, we obtain

$$
\begin{aligned}
\left\|I I I_{3}\right\| \leq & C \int_{R^{n}}\left(\iint_{R_{+}^{n+1}} \frac{\left[\psi_{t}(y-z)|| f_{2}(z)|| R_{m}\left(\tilde{A} ; x_{0}, z\right) \mid\right.}{\left|x_{0}-z\right|^{m}}\right. \\
& \left.\left.\times\left|\chi_{\Gamma(x)}(y, t)-\chi_{\Gamma\left(x_{0}\right)}(y, t)\right|\right]^{2} \frac{d y d t}{t^{n+1}}\right)^{1 / 2} d z \\
\leq & C \int_{R^{n}} \frac{\left|f_{2}(z)\right|\left|R_{m}\left(\tilde{A} ; x_{0}, z\right)\right|}{\left|x_{0}-z\right|^{m}} \\
& \times\left|\iint_{\Gamma(x)} \frac{t^{1-n} d y d t}{(t+|y-z|)^{2 n+2}}-\iint_{\Gamma\left(x_{0}\right)} \frac{t^{1-n} d y d t}{(t+|y-z|)^{2 n+2}}\right|^{1 / 2} d z \\
\leq & C \int_{R^{n}} \frac{\left|f_{2}(z)\right|\left|R_{m}\left(\tilde{A} ; x_{0}, z\right)\right|}{\left|x_{0}-z\right|^{m}} \\
& \times\left(\iint_{|y| \leq t \mid}^{\mid} \frac{1}{(t+|x+y-z|)^{2 n+2}}-\frac{1}{\left(t+\left|x_{0}+y-z\right|\right)^{2 n+2}} \mid \frac{d y d t}{t^{n-1}}\right)^{1 / 2} d z \\
\leq & C \int_{R^{n}} \frac{\left|f_{2}(z)\right|\left|R_{m}\left(\tilde{A} ; x_{0}, z\right)\right|}{\left|x_{0}-z\right|^{m}}\left(\iint_{|y| \leq t} \frac{\left|x-x_{0}\right| t^{1-n} d y d t}{(t+|x+y-z|)^{2 n+3}}\right)^{1 / 2} d z
\end{aligned}
$$




$$
\begin{aligned}
& \leq C \int_{R^{n}} \frac{\left|f_{2}(z)\right|\left|x-x_{0}\right|^{1 / 2}\left|R_{m}\left(\tilde{A} ; x_{0}, z\right)\right|}{\left|x_{0}-z\right|^{m+n+1 / 2}} d z \\
& \leq C \sum_{|\alpha|=m}\left\|D^{\alpha} A\right\|_{B M O} \sum_{k=1}^{\infty} 2^{-k / 2} \frac{1}{\left|2^{k} \tilde{Q}\right|} \int_{2^{k} \tilde{Q}}|f(z)| d z \\
& \leq C \sum_{|\alpha|=m}\left\|D^{\alpha} A\right\|_{B M O} M(f)(\tilde{x}) ;
\end{aligned}
$$

For $I I_{4}$, similar to the proof of $I I_{1}$ and $I I_{3}$, we get

$$
\begin{aligned}
\left\|I I I_{4}\right\| & \leq C \sum_{|\alpha|=m} \int_{R^{n}}\left(\frac{\left|x-x_{0}\right|}{\left|x_{0}-z\right|^{n+1}}+\frac{\left|x-x_{0}\right|^{1 / 2}}{\left|x_{0}-z\right|^{n+1 / 2}}\right)\left|D^{\alpha} \tilde{A}(y)\right|\left|f_{2}(z)\right| d z \\
& \leq C \sum_{|\alpha|=m} \sum_{k=1}^{\infty}\left(2^{-k}+2^{-k / 2}\right) \frac{1}{\left|2^{k} \tilde{Q}\right|} \int_{2^{k} \tilde{Q}}|f(z)|\left|D^{\alpha} A(z)-\left(D^{\alpha} A\right)_{\tilde{Q}}\right| d z \\
& \leq C \sum_{|\alpha|=m} \sum_{k=1}^{\infty} k\left(2^{-k}+2^{-k / 2}\right)\left(\left\|D^{\alpha} A\right\|_{\exp L, 2^{k}} \tilde{Q}\|f\|_{L \log L, 2^{k} \tilde{Q}}\right. \\
& \left.\quad+\left\|D^{\alpha} A\right\|_{B M O} M(f)(\tilde{x})\right) \\
& \leq C \sum_{|\alpha|=m} \sum_{k=1}^{\infty} k\left(2^{-k}+2^{-k / 2}\right)\left\|D^{\alpha} A\right\|_{B M O} M_{L \log L}(f)(\tilde{x}) \\
& \leq C \sum_{|\alpha|=m}\left\|D^{\alpha} A\right\|_{B M O} M^{2}(f)(\tilde{x}) .
\end{aligned}
$$

Thus,

$$
I I I \leq C \sum_{|\alpha|=m}\left\|D^{\alpha} A\right\|_{B M O} M^{2}(f)(\tilde{x}) .
$$

This completes the proof of Theorem 1 .

From Theorem 1 and the weighted boundedness of $S_{\psi}$ and $M$, we may obtain the conclusion of Theorem 2 .

From Theorem 1 and Lemma 2, we may obtain the conclusion of Theorem 3.

\section{REFERENCES}

[1] J. CoHEN, A sharp estimate for a multilinear singular integral on $R^{n}$, Indiana Univ. Math. J. 30 (1981), 693-702.

[2] J. Cohen ANd J. Gosselin, On multilinear singular integral operators on $R^{n}$, Studia Math. 72 (1982), 199-223.

[3] J. Cohen AND J. Gosselin, A BMO estimate for multilinear singular integral operators, Illinois J. Math. 30 (1986), 445-465. 
[ 4 ] Y. Ding, A note on multilinear fractional integrals with rough kernel, Adv. in Math. (China) 30 (2001), 238-246.

[5] Y. Ding AND S. Z. Lu, Weighted Boundedness for a class of rough multilinear operators, Acat Math. Sinica, (China) 17 (2001), 517-526.

[6] G. B. Folland and E. M. Stein, Hardy spaces on homogenous groups, Princeton Univ. Press, Princeton, NJ, 1982.

[7] J. Garcia-Cuerva and J. L. Rubio de Francia, Weighted norm inequalities and related topics, North-Holland Math. Stud. 16, Amsterdam, 1985.

[ 8 ] G. Hu AND D. C. YANG, A variant sharp estimate for multilinear singular integral operators, Studia Math. 141 (2000), 25-42.

[9] L. Z. LiU, Weighted weak type estimates for commutators of Littlewood-Paley operator, Japanese J. of Math. 29(1) (2003), 1-16.

[10] C. Perez, Endpoint estimate for commutators of singular integral operators, J. Func. Anal. 128 (1995), 163-185.

[11] C. Perez, Weighted norm inequalities for singular integral operators, J. London Math. Soc. 49 (1994), 296-308.

[12] A. Torchinsky, The real variable methods in harmonic analysis, Pure and Applied Math. 123, Academic Press, New York, 1986.

Department of Mathematics

Changsha University of Science and Technology

Changsha, 410077

P. R. CHINA

e-mail: lanzheliu@263.net 